\title{
Remdesivir and Lopinavir/Ritonavir as Potential Drugs to Treat Corona Virus Disease 2019
}

\author{
Bzikha Reda*, Bouhmou Ayoub, Bzikha Ilham, Bouchnafati Sanaa \\ Department of Medicine and Pharmacy, Sidi Mohamed Ben Abdellah University, Fez, Morocco
}

\begin{abstract}
Drugs that are efficacious against SARS-CoV-2 have yet to be established. Remdesivir and Lopinavir/ritonavir have garnered considerable attention for their potential to treat coronavirus disease 2019 (COVID-19). Remdesivir not only in vivo but also in vitro testing shows the inhibition of human coronavirus replication, including SARS-CoV as well as Lopinavir/ritonavir that shows promising antiviral drug against SARS-CoV-2.
\end{abstract}

Keywords: Remdesivir; Lopinavir; Ritonavir; COVID-19; SARS-CoV-2

\section{INTRODUCTION}

Drugs that are efficacious against SARS-CoV-2 have yet to be established. Remdesivir and Lopinavir/ritonavir have garnered considerable attention for their potential to treat coronavirus disease 2019 (COVID-19).

\section{REMDESIVIR}

Remdesivir is a 1 cyano substituted adenosine analogue prodrug with broad antiviral activity, initially used against Ebola and it has been shown to inhibit SARS-CoV-2 [1,2]. Its antiviral mechanism is due its incorporation into viral RNA chains resulting premature termination, Remdesivir has been recently recognized as a promising antiviral drug against RNA viruses, especially 2019-nCoV as it been showed by preliminary data in various studies $[3,4]$.

Remdesivir, as a prodrug with a broad-spectrum antiviral activity against coronaviruses, was recently confirmed to inhibit 2019-nCoV in vitro [5-8]. Not only in vivo but also in vitro testing this drug shows the inhibition of human coronavirus replication, including SARSCoV $[5,7,8]$. Therefore, Remdesivir was initially only available for compassionate use but received orphan drug status from the US Food and Drug Administration on March 23, 2020.

Thus, Remdesivir is currently evaluated in phase 4 clinical trials for SARS-CoV-2, in the United States, South Korea, China, and Europe. Two of on-going multicentre ones are evaluating this drug in hospitalized patients with moderate or severe symptoms and it showed reduction of pneumonia associated symptoms of some COVID-19 patients $[9,10]$. Remdesivir is generally well tolerated but hepatotoxicity and nephrotoxicity has been observed as it showing in a recent study that promises its antiviral activity against SARS-CoV-2.

\section{LOPINAVIR/RITONAVIR}

Besides Remdesivir, Lopinavir/ritonavir showed promising antiviral drug against SARS-CoV-2 [11,12]. Lopinavir/ritonavir is generally used in the treatment for HIV infection by suppressing the replication of HIV-1 [13]. In vitro Lopinavir/ritonavir demonstrated activity against coronaviruses via inhibition of 3-chy-motrypsin-like protease, this drug was found to inhibit SARS-CoV-2 replication in vitro $[13,14]$. Some clinical studies in SARS were associated with reduced mortality and intubation rates [11,12]. Lopinavir/ ritonavir can also reduce pneumonia-associated symptoms of some COVID-19 patients $[15,16]$. That is proven in other study that it can significantly lead to the reduction of acute respiratory distress syndrome caused by SARS infection [17].

In a clinical study, the therapeutic effect of Lopinavir/ritonavir combined therapy was better than that of the therapy without Lopinavir/ritonavir, no adverse effects in liver toxic and side effects compared with the control group after the combination treatment with Lopinavir/ritonavir and adjuvant drugs. However, some data suggest that Arbidol monotherapy is more effective than Lopinavir/ ritonavir in treating COVID-19, and indicate that Arbidol monotherapy may be superior to Lopinavir/ritonavir in treating COVID-19. The most commonly used and studied Lopinavir/ ritonavir dosing regimen for COVID-19 treatment is $400 \mathrm{mg} / 100$ mg twice daily for up to 14 days with or without ribavirin as is it recommended by China National Health Commission, 2020

Correspondence to: Bzikha Reda, Department of Medicine and Pharmacy, Sidi Mohamed Ben Abdellah University, Fez, Morocco, E-mail: redabzikha@ gmail.com

Received: October 24, 2020; Accepted: November 07, 2020; Published: November 14, 2020

Citation: Reda B, Ayoub B, Ilham B, Sanaa B (2020) Remdesivir and Lopinavir/Ritonavir as Potential Drugs to Treat Corona Virus Disease 2019. J Antivir Antiretrovir. S6.002

Copyright: ( 2020 Reda B, et al. This is an open-access article distributed under the terms of the Creative Commons Attribution License, which permits unrestricted use, distribution, and reproduction in any medium, provided the original author and source are credited. 
[18,19].

Combinational therapy of Lopinavir with the other effective compounds against SARS-CoV-2 virus may rise synergy and reduce adverse effects of Lopinavir/ritonavir that include gastrointestinal distress and hepatotoxicity [20]. Another study proved that the combination treatment of Lopinavir/ritonavir and routine adjuvant medicine against pneumonia could produce much better efficacy on patients with COVID-19 infection compared to treatment with adjuvant medicine alone [21].

The $7^{\text {th }}$ edition of the novel coronavirus diagnosis and treatment plan published by the National Health Commission of the People's Republic of China, options for antiviral therapy include aerosolized $\alpha$ interferon, Lopinavir/ritonavir, ribavirin in combination with Lopinavir/ritonavir, chloroquine phosphate, or Arbidol [22].

\section{CONCLUSION}

Remdesivir and Lopinavir/Ritonavir are highly effective in the control of 2019-nCoV infection in vitro as in vivo. Since these compounds have been used in human patients with a safety track record, we suggest that they should be used in human patients suffering from the novel coronavirus disease.

\section{REFERENCES}

1. Wit E, Feldmann F, Cronin J. Prophylactic and therapeutic remdesivir (GS-5734) treatment in the rhesus macaque model of MERS-CoV infection. Proc Natl Acad Sci. 2020; 117(12):6771-6776.

2. Mulangu S, Dodd LE, Davey RT. A randomized, controlled trial of Ebola virus disease therapeutics. N Engl J Med. 2019; 381(24):22932303.

3. Mulangu S, Dodd LE, Davey RT, Mbaya OT, Proscham M.Randomized, controlled trial of Ebola Virus disease therapeutics. N Engl J Med. 2019; 381:2293-2303.

4. Zhou P. A pneumonia outbreak associated with a new coronavirus of probable bat origin nature. 2020; 270-273.

5. Wang M, Cao R, Zhang L. Remdesivir and chloroquine effectively inhibit the recently emerged novel coronavirus (2019-nCoV) in vitro. Cell Res. 2020; 30(3):269-271.

6. Brown AJ, Won JJ, Graham RL, Dinnon KH, Sims AC. Broad spectrum antiviral remdesivir inhibits human endemic and zoonotic deltacoronaviruses with a highly divergent RNA dependent RNA polymerase. Antivir Res. 2019; 169:104541.

7. Sheahan TP, Sims AC, Leist SR. Comparative therapeutic efficacy of remdesivir and combination lopinavir, ritonavir, and interferon beta against MERS-CoV. Nat Commun. 2020; 11(1).
8. Wit E, Feldmann F, Cronin J. Prophylactic and therapeutic remdesivir (GS-5734) treatment in the rhesus macaque model of MERS-CoV infection. Proc Natl Acad Sci. 2020; 201922083.

9. Wiggins BS, Dixon DL, Neyens RR, Page RL, Gluckman TJ. Select Drug-Drug Interactions With Direct Oral Anticoagulants: JACC Review Topic of the Week. J Am Coll Cardiol. 2020; 75(11):13411350.

10. Chauvin B, Drouot S, Barrail-Tran A, Taburet AM. Drug-drug interactions between HMG-CoA reductase inhibitors (statins) and antiviral protease inhibitors. Clin Pharmacokinet. 2013; 52(10):815 831.

11. Yao TT, Qian JD, Zhu WY, Wang Y, Wang GQ. A systematic review of lopinavir therapy for SARS coronavirus and MERS coronavirus-A possible reference for coronavirus disease-19 treatment option. J Med Virol. 2020.

12. Chan KS, Lai ST, Chu CM. Treatment of severe acute respiratory syndrome with lopinavir/ritonavir: A multicentre retrospective matched cohort study. Hong Kong Med J. 2003; 9(6):399-406.

13. Wilde AH, Jochmans D, Posthuma CC. Screening of an FDAapproved compound library identifies four small-molecule inhibitors of Middle East respiratory syndrome coronavirus replication in cell culture. Antimicrob Agents Chemother. 2014; 58(8):4875-4884.

14. Chu CM, Cheng VC, Hung IF. HKU/UCH SARS Study Group. Role of lopinavir/ritonavir in the treatment of SARS: initial virological and clinical findings. Thorax. 2004; 59(3):252-256.

15. Holshue ML, DeBolt C, Lindquist S, Lofy KH, Wiesman J. First case of 2019 novel coronavirus in the United States. N Engl J Med. 2020; 382(10):929-936.

16. Wang Z, Chen X, Lu Y, Chen F, Zhang W. Clinical characteristics and therapeutic procedure for four cases with 2019 novel coronavirus pneumonia receiving combined Chinese and Western medicine treatment. Biosci Trends. 2020; 14 (1):64-68.

17. Chu C. HKU/UCH SARS Study Group. Thorax. 2004; 59:252-256.

18. National Health Commission and State Administration of Traditional Chinese Medicine. Diagnosis and treatment protocol for novel coronavirus pneumonia. 2020.

19. Cao B, Wang Y, Wen D. A trial of lopinavir-ritonavir in adults hospitalized with severe COVID-19. N Engl J Med. 2020.

20. Lopinavir/ritonavir. Hudson. 2016.

21. Ye XT, Luo YL, Xia SC. Clinical efficacy of lopinavir/ritonavir in the treatment of Coronavirus disease 2019. Eur Rev Med Pharmacol Sci. 2020;24(6):3390-3396

22. WHO. Summary of probable SARS cases with onset of illness from 1 November 2002 to 31 July 2003. 2003 